\title{
Another Breast Cancer Entity Confirmed: Genomics of Invasive Lobular Breast Cancer
}

\author{
Michael L. Gatza, Rutgers, The State University of New Jersey, New Brunswick, NJ \\ Lisa A. Carey, University of North Carolina at Chapel Hill, Chapel Hill, NC
}

See accompanying article on page 1872

Invasive lobular carcinoma (ILC) accounts for up to $15 \%$ of all invasive breast cancers. ${ }^{1}$ We have long recognized the unique clinical characteristics of ILC, but an increased understanding of its biology in contrast with the more common breast cancer histologic type, infiltrating ductal carcinoma (IDC), has emerged. The article that accompanies this editorial by Desmedt et $\mathrm{al}^{2}$ reinforces and extends this understanding.

ILC is characterized by loss of normal cell-cell adhesion, which results in discohesive cells that fail to form glandular structures. The defining genetic feature that distinguishes ILC from IDC is CDH1 mutation or loss of either mRNA or protein expression of the cell adhesion molecule E-cadherin; 95\% of ILCs demonstrate some form of E-cadherin alteration. ${ }^{3,4}$ Most ILCs are of the classical histologic type, which is low grade and possesses a low to intermediate mitotic index, ${ }^{1}$ whereas less common ILC variants, such as the solid and mixed nonclassical types, often are high grade and associated with a poorer outcome than classical ILC. ${ }^{5}$ ILC of all types usually expresses estrogen receptors (ERs) and progesterone receptors and are rarely clinically human epidermal growth factor receptor 2 (HER2) positive. Perhaps related to $C D H 1$ loss, which in other tumor types confers increased risk of invasion and metastasis, 6,7 some studies have suggested that patients with ILC have similar or worse long-term outcomes compared with patients with stagematched IDC. ${ }^{8,9}$ Metastatic ILC also has unusual patterns of spread to sites such as the ovaries, lining of the GI tract, and peritoneum. ${ }^{1,8}$ All these features are consistent with a unique biology.

Despite many studies that investigated the genomic and mutational landscape of breast cancer, lobular breast tumors have been largely underrepresented. ${ }^{4,10}$ Several studies have examined transcriptional profile differences that distinguish ILC from IDC; however, with the exception of the loss of $C D H 1$ expression, few ILC-distinguishing features have been identified. ${ }^{11-16}$ Desmedt et $\mathrm{al}^{2}$ genomically profiled the largest cohort of primary invasive lobular breast tumors analyzed to date, which included 430 tumors analyzed for mutations and 170 for copy number aberrations, to identify and characterize the recurrent genomic alterations present in these tumors. The results of this large study in conjunction with remarkably consistent findings from 127 ILCs and 490 IDCs in The Cancer Genome Atlas (TCGA) project ${ }^{3}$ and 144 ILCs from a European cohort ${ }^{17}$ have clear biologic and clinical implications with regard to genetic alterations that drive lobular breast cancer.
The most frequent significantly altered gene identified in this study was $\mathrm{CDH} 1$ in which loss-of-function mutations occurred in $65 \%$ of cases and loss of heterozygosity or deletion was identified in $94 \%$ of patients. These findings are consistent with other studies and underscore the role of E-cadherin loss in contributing to the characteristic discohesive morphology associated with ILC. ${ }^{3}$

Given that the majority of ILCs are hormone receptor positive, endocrine therapy is key to treatment. ILC appears to derive particular benefit from treatment with aromatase inhibitors compared with tamoxifen, which is possibly related to relative tamoxifen resistance. ${ }^{18,19}$ The current study and TCGA analysis ${ }^{3}$ identified alterations that affect ER signaling, including mutations in ER modulators that could lead to altered or divergent ER-mediated signaling. Of note, the authors found that focal copy number gains at 6q25.1, including ESR1, occur in 25\% of lobular tumors and that copy number changes corresponded to increased expression of an ESR1 mRNA gene expression signature. Although TCGA analysis did not indicate copy number gain as significantly enriched in ILC, it revealed increased ER activity in the form of significantly higher levels of total and phosphorylated ER protein. Beyond ESR1 and ER expression, ILC-specific mutations that affect ER cofactors were identified. In both TCGA and the current study, ILC was characterized by an increased incidence of FOXA1 mutations, whereas IDC was enriched for GATA3 mutations. Similarly, lower GATA3 mRNA and protein expression in ILC and reduced methylation at ER promoters were observed in the TCGA cohort. ${ }^{3}$ Because both GATA3 and FOXA1 modulate ER transcriptional activity, the results suggest preferential binding of ER to FOXA1-bound promoter sites in ILC and to GATA3-bound sites in IDC, which could lead to differential ESR1-regulated gene expression. ${ }^{3,20}$ Both altered ER protein expression and unique mutation patterns in ER transcription complex family members can affect response to endocrine therapy.

Beyond $\mathrm{CDH} 1$ and ER signaling, 50\% of ILCs harbor mutations in one of three key genes that regulate the phosphatidylinositol 3-kinase (PI3K) pathway. Specifically, Desmedt et $\mathrm{al}^{2}$ identified an increased frequency of lobular-specific mutations in PIK3CA, the PI3K-negative regulator PTEN, and the downstream kinase $A K T 1$. An increased incidence of PTEN loss-of-function mutations or loss of heterozygosity as well as a high frequency of 
PIK3CA mutations in ILC was observed in the TCGA and European studies. ${ }^{3,17}$ In TCGA, loss or mutation of PTEN corresponded with decreased PTEN protein expression and a concomitant increase in activated or phosphorylated Akt protein expression in lobular tumors. In fact, the levels of phosphorylated Akt protein expression identified in lobular tumors was comparable to the expression of these proteins in basal-like or HER2-enriched ductal tumors, which have been shown to have the highest levels of PI3K/Akt signaling among IDC tumors. ${ }^{4}$ Beyond protein expression, integrative analysis of TCGA genomic data identified multiple alterations that contribute to aberrant Akt/mammalian target of rapamycin signaling in $45 \%$ of ILC cases. Previous studies have linked loss of E-cadherin with Akt activation and epidermal growth factor receptor (EGFR) expression. Results from TCGA that demonstrated overexpression of phosphorylated EGFR in conjunction with data that describe the increased incidence of PI3K/Akt pathway mutations from both the current study and TCGA suggest that the ILC tumor environment provides a favorable cellular context for altered Akt signaling $3,4,21,22$ and that the targeting of the PI3K/Akt pathway may be particularly relevant in ILC.

The study by Desmedt et $\mathrm{al}^{2}$ is the first to characterize HER2 and HER3 mutations in ILC. Overall, $14 \%$ of ILC tumors were either HER2 amplified or carried a mutation in HER2 or HER3. These data suggest that ILC has the highest incidence of HER2mutated, nonamplified tumors among all breast cancer subsets. In the TCGA study, 10\% of ILCs had amplified or mutated HER2, and reverse phase protein assays revealed increased EGFR activity in ILC. Together, these studies suggest that $E R B B$ family signaling may significantly contribute to ILC biology. Additional analyses are necessary to fully illuminate the context and extent of alterations that affect this family of genes as well as to understand the clinical relevance of these alterations.

In the TCGA and European studies, ${ }^{3,17}$ transcriptome analyses found at least two molecularly distinct subsets of ILC, including an immune-related subset that appeared to have both high expression of lymphocyte signaling pathway components and negative immune response regulators. This finding may have consequences for the use of immune checkpoint inhibitors or other immune-based approaches in ILC.

Desmedt et al, ${ }^{2}$ in concert with similar initiatives published within the past few months, ${ }^{3}$ have extensively characterized the genomic landscape of ILC and have produced remarkably consistent results that identify at least three significantly altered and therapeutically relevant pathways. These are an important first step to understanding ILC as well as the potential treatment implications of this unique biologic subset of breast cancer. It is now clear that ILC and IDC are distinct molecular diseases, a fact that should be considered in future experimental and therapeutic initiatives.

\section{AUTHORS' DISCLOSURES OF POTENTIAL CONFLICTS OF INTEREST}

Disclosures provided by the authors are available with this article at www.jco.org.

\section{REFERENCES}

1. McCart Reed $A E$, Kutasovic JR, Lakhani SR, et al: Invasive lobular carcinoma of the breast: Morphology, biomarkers and 'omics. Breast Cancer Res 17:12, 2015

2. Desmedt C, Zoppoli G, Gundem G: Genomic characterization of primary invasive lobular breast cancer. J Clin Oncol 34:1872-1881, 2016

3. Ciriello G, Gatza ML, Beck $A H$, et al: Comprehensive molecular portraits of invasive lobular breast cancer. Cell 163:506-519, 2015

4. Cancer Genome Atlas Network: Comprehensive molecular portraits of human breast tumours. Nature 490:61-70, 2012

5. Iorfida M, Maiorano $E$, Orvieto $E$, et al: Invasive lobular breast cancer: Subtypes and outcome. Breast Cancer Res Treat 133:713-723, 2012

6. Brinck $U$, Jacobs $S$, Neuss $M$, et al: Diffuse growth pattern affects E-cadherin expression in invasive breast cancer. Anticancer Res 24:2237-2242, 2004

7. Richards FM, McKee SA, Rajpar $\mathrm{MH}$, et al: Germline E-cadherin gene $(\mathrm{CDH} 1)$ mutations predispose to familial gastric cancer and colorectal cancer. Hum Mol Genet 8:607-610, 1999

8. Arpino G, Bardou VJ, Clark GM, et al: Infiltrating lobular carcinoma of the breast: Tumor characteristics and clinical outcome. Breast Cancer Res 6: R149-R156, 2004

9. Pestalozzi BC, Zahrieh D, Mallon E, et al: Distinct clinical and prognostic features of infiltrating lobular carcinoma of the breast: Combined results of 15 International Breast Cancer Study Group clinical trials. J Clin Oncol 26:3006-3014, 2008

10. Curtis C, Shah SP, Chin SF, et al: The genomic and transcriptomic architecture of 2,000 breast tumours reveals novel subgroups. Nature 486:346-352, 2012

11. Bertucci $F$, Orsetti $B$, Nègre $V$, et al: Lobular and ductal carcinomas of the breast have distinct genomic and expression profiles. Oncogene 27:5359-5372, 2008

12. Gruel N, Lucchesi $C$, Raynal V, et al: Lobular invasive carcinoma of the breast is a molecular entity distinct from luminal invasive ductal carcinoma. Eur $\mathrm{J}$ Cancer 46:2399-2407, 2010

13. Korkola JE, DeVries S, Fridlyand J, et al: Differentiation of lobular versus ductal breast carcinomas by expression microarray analysis. Cancer Res 63: 7167-7175, 2003

14. Turashvili G, Bouchal J, Baumforth $K$, et al: Novel markers for differentiation of lobular and ductal invasive breast carcinomas by laser microdissection and microarray analysis. BMC Cancer 7:55, 2007

15. Weigelt B, Geyer FC, Natrajan R, et al: The molecular underpinning of lobular histological growth pattern: A genome-wide transcriptomic analysis of invasive lobular carcinomas and grade- and molecular subtype-matched invasive ductal carcinomas of no special type. J Pathol 220:45-57, 2010

16. Zhao $H$, Langerød $A$, Ji $Y$, et al: Different gene expression patterns in invasive lobular and ductal carcinomas of the breast. Mol Biol Cell 15:2523-2536, 2004

17. Michaut M, Chin SF, Majewski I, et al: Integration of genomic, transcriptomic and proteomic data identifies two biologically distinct subtypes of invasive lobular breast cancer. Sci Rep 6:18517, 2016

18. Metzger Filho $O$, Giobbie-Hurder $A$, Mallon $E$, et al: Relative effectiveness of letrozole compared with tamoxifen for patients with lobular carcinoma in the BIG 1-98 trial. J Clin Oncol 33:2772-2779, 2015

19. Sikora MJ, Cooper KL, Bahreini A, et al: Invasive lobular carcinoma cell lines are characterized by unique estrogen-mediated gene expression patterns and altered tamoxifen response. Cancer Res 74:1463-1474, 2014

20. Theodorou $V$, Stark $R$, Menon $S$, et al: GATA3 acts upstream of FOXA1 in mediating ESR1 binding by shaping enhancer accessibility. Genome Res 23:12-22, 2013

21. Lau MT, Klausen $C$, Leung $P C$ : E-cadherin inhibits tumor cell growth by suppressing PI3K/Akt signaling via $\beta$-catenin-Egr1-mediated PTEN expression. Oncogene 30:2753-2766, 2011

22. Liu $X, S u L$, Liu $X$ : Loss of $\mathrm{CDH}_{1}$ up-regulates epidermal growth factor receptor via phosphorylation of $Y B X 1$ in non-small cell lung cancer cells. FEBS Lett 587:3995-4000, 2013

DOI: 10.1200/JCO.2015.66.3872; published online ahead of print at www.jco.org on March 28, 2016. 


\section{AUTHORS' DISCLOSURES OF POTENTIAL CONFLICTS OF INTEREST}

Another Breast Cancer Entity Confirmed: Genomics of Invasive Lobular Breast Cancer

The following represents disclosure information provided by authors of this manuscript. All relationships are considered compensated. Relationships are self-held unless noted. I = Immediate Family Member, Inst = My Institution. Relationships may not relate to the subject matter of this manuscript. For more information about ASCO's conflict of interest policy, please refer to www.asco.org/rwc or jco.ascopubs.org/site/ifc.

\section{Michael L. Gatza}

No relationship to disclose
Lisa A. Carey

Research Funding: GlaxoSmithKline, Genentech 